\title{
NO-sensitive guanylyl cyclase: Identification and purification of the dimerization domain
}

\author{
Corina Wagner*, Michael Russwurm and Doris Koesling
}

\author{
Address: Institut für Pharmakologie und Toxikologie, Medizinische Fakultät, MA N1, Ruhr-Universität Bochum, 44780 Bochum, Germany \\ Email: Corina Wagner* - Corina.Wagner@ruhr-uni-bochum.de \\ * Corresponding author
}

from 2nd International Conference of cGMP Generators, Effectors and Therapeutic Implications

Potsdam, Germany, 10-12 June, 2005

Published: 16 June 2005

BMC Pharmacology 2005, 5(SuppI I):P57 doi:I0.1186/147I-2210-5-SI-P57

The nitric oxide (NO)-sensitive guanylyl cyclase plays a key role in diverse signalling pathways by catalysing the biosynthesis of the messenger molecule cGMP. To date, two isoforms of the enzyme $\left(\alpha_{1} \beta_{1}\right.$ and $\left.\alpha_{2} \beta_{1}\right)$ are known, both of which contain a prosthetic heme group bound to the histidine 105 of the $\beta_{1}$ subunit. The $\alpha_{1} \beta_{1}$ isoform is ubiquitously expressed and is considered to be soluble, whereas the $\alpha_{2} \beta_{1}$ isoform is mainly expressed in brain and is located to the membrane via interaction with PSD-95. The three-dimensional structure of NO-sensitive guanylyl cyclase has not been solved. Yet, by sequence comparison the subunits are generally divided into three domains. An $\mathrm{N}$-terminal regulatory domain, a central domain postulated to be involved in dimerization and a C-terminal catalytic domain. For catalytic activity dimerization of the $\alpha$ and $\beta$ subunit is mandatory, but until recently the regions involved in the interaction were unknown.

By coprecipitation of several deletion mutants of the $\alpha_{1}$ and $\beta_{1}$ subunit, expressed in Sf9-cells, we have mapped the $\alpha_{1}$ dimerization domain to amino acids 61-462. Within this region, we identified two binding sites. One, as postulated, covering the central amino acids 367-462, the other, quite unexpectedly, located in the $\mathrm{N}$ terminus of the $\alpha_{1}$-subunit (amino acids 61-128). By itself neither region was sufficient to mediate full dimerization. Of the $\beta_{1}$ subunit the $\mathrm{N}$-terminal and central amino acids 1-385 exhibited wt-like binding to the identified $\alpha_{1}$ domain. To further characterize the short, but stable, dimeric NO-sensitive guanylyl cyclase we purified the mutant from Sf9cells and analysed heme-coordination and binding of $\mathrm{NO}$ in spectrophotometric measurements. The dimeric state of the mutant was confirmed by gelfiltration analysis.
As $\beta_{1}$ is the binding partner for both the $\alpha_{1}$ and $\alpha_{2}$ subunit, we reasoned that dimerization of the $\alpha_{2} \beta_{1}$ isoform must be mediated by parts of the $\alpha_{2}$ subunit homologous to the $\alpha_{1}$ dimerization domain. Accordingly, we cloned the respective sequences of $\alpha_{2}$ (amino acids 76-501) and analysed binding of the mutant to the $\mathrm{N}$-terminal and central regions of $\beta_{1}$. 